\title{
The effect of postoperative radiotherapy on the survival of patients with resectable stage III-N2 non-small-cell lung cancer: a systematic review and meta-analysis
}

\author{
H. ZHANG ${ }^{1}$, D. X. ZHANG ${ }^{2}$, T. JU², J. ZHOU ${ }^{1, *}$ \\ ${ }^{1}$ Center for Hematology and Oncology, The First Affiliated Hospital, Harbin Medical University, Harbin, Heilongjiang, China; ${ }^{2}$ Department of \\ Oncology, The First Affiliated Hospital, Harbin Medical University, Harbin, Heilongjiang, China \\ *Correspondence: zhoujinHMU@21cn.com
}

Received December 13, 2018 / Accepted March 13, 2019

\begin{abstract}
In the potentially resectable cases of stage III-N2 non-small-cell lung cancer (NSCLC), the optimal post-operative treatment regimen for these patients is uncertain and post-operative radiation therapy (PORT) with chemotherapy is typically recommended. Our aim was to reassess the data of PORT on overall survival (OS) and disease-free survival (DFS) in stage III-N2 NSCLC, in order to figure out whether PORT might lead to a moderate improvement in local control and survival besides resection and adjuvant chemotherapy. A comprehensive search strategy was performed in EMBASE, PubMed, and Cochrane Library for relevant studies comparing PORT combined with adjuvant chemotherapy or adjuvant chemotherapy alone on OS and DFS in resectable stage III-N2 NSCLC. Data were extracted to estimate the effects of PORT on OS and DFS. Eleven studies with 8,928 patients were included. This meta-analysis demonstrated a trend in improving OS associated with the use of PORT (HR=0.88; 95\% CI, 0.76 to $1.03 ; \mathrm{p}=0.11)$ and a significantly difference of effect on DFS associated with the use of PORT $(\mathrm{HR}=0.78 ; 95 \% \mathrm{CI}, 0.66$ to $0.92 ; \mathrm{p}=0.003)$. In a subgroup analysis on Caucasian patients, there was a statistically significant benefit $(\mathrm{HR}=0.88 ; 95 \% \mathrm{CI}, 0.81$ to $0.96 ; \mathrm{p}=0.003)$ on OS for PORT. Our findings demonstrate that in the postoperative treatment for patients with stage III-N2 NSCLC, PORT is associated with improved OS and leads to a significantly increased DFS.
\end{abstract}

Key words: non-small-cell lung cancer, stage III-N2, postoperative radiotherapy, overall survival, disease-free survival

Despite advances in tobacco control and multimodality treatment in the past few decades, lung cancer remains a major cause of cancer-related mortality in most countries, over $80 \%$ of which is of non-small cell cancer (NSCLC), and approximately $35 \%$ of these patients afflicted with locally advanced non-metastatic disease (stage III) [1]. Surgery offers the only potential cure for patients suffered from NSCLC, although only about one-fifth of the patients are eligible for radical resection, and the postsurgical survival rate of patients with stage III NSCLC is extremely poor [2]. Since the 1960s, adjuvant chemotherapy has been applied for treating NSCLC, which is proved effective and is considered as a standard treatment option following surgically resected (Stage IB ( $>4 \mathrm{~cm}$ )-IIIA) NSCLC presently [3]. External beam radiation therapy can be taken into account for multimodality treatment of resectable stage III NSCLC, whether administered before or after surgical resection, however, conflicting and inconclusive results make the application of radiotherapy elusive [4]. For those patients who have received combination modality approach, including chemotherapy, radiation, and/or surgery, the current 5-year survival rate for stage III NSCLC is approximately $20-25 \%$ [5, 6]. Many patients already have an advanced form of NSCLC when they are first diagnosed, for patients with clinically or pathologically staged III-N2 operable NSCLC, there is still a considerable debate among many organizations and cooperative groups regarding the best strategy of comprehensive treatment [7]. As a consequence, the standard treatment options for patients with resectable stage III-N2 NSCLC remain controversial.

In most cases of stage III-N2 NSCLC, concurrent or sequential chemoradiotherapy depending on patients' physical status is considered as a standard treatment. Alternatively, for patients with potentially resectable stage III-N2 NSCLC, a surgical multimodality treatment can be offered in an attempt to improve survival. The optimal postoperative 
treatment regimen for these patients is uncertain, whereas post-operative radiation therapy (PORT) with chemotherapy is typically recommended. In $\mathrm{pN} 2$ patients having received complete resection and adjuvant chemotherapy, the risk of locoregional relapse is still $20-40 \%[8,9]$, emphasizing the need for additional therapy to improve locoregional control. Several trials have been conducted to investigate the role of PORT in NSCLC, of which the results revealed improved local control and a trend towards improved disease-free survival (DFS), but no overall survival (OS) benefit $[10,11]$. A large retrospective population-based cohort study based on 7,465 patients from Surveillance, Epidemiology and End Results databases found that PORT was associated with a significant increase in survival in patients with N2 NSCLC. Of note, in this study, the use of PORT impaired survival in patients with N0 and N1 disease [12]. Another retrospective study based on 3,395 patients from the National Cancer Data Base indicated that PORT utilization trend to improve overall survival across all nodal stages in patients with incompletely resected stage II or III N0-2 NSCLC [13]. Despite the inconsonant results of impaired survival in $\mathrm{N} 0$ and $\mathrm{N} 1$, possible benefit of PORT in resectable N2 NSCLC was confronted with challenges. A meta-analysis based on individual patient data of 2,128 participants from 9 trials showed a significantly adverse effect of PORT on OS with an 7\% lower survival rate at two years ( $48 \%$ vs. $55 \%$ ) in patients with completely resected NSCLC, but did trend a possible benefit of survival in the pN2 subgroup [14]. Following update of this metaanalysis based on individual patient data of 2,343 participants from 11 trials demonstrated PORT decreased the survival rate at two years by $6 \%$ (53\% vs. $58 \%)$ of the whole patient group, and the newest update published in 2010 with new trials came to the same conclusion $[15,16]$.

Many phase III trials have proven the advantageous effect of adjuvant chemotherapy [17, 18]. In contrast with the consistent conclusions of adjuvant chemotherapy, whether PORT or not remains controversial. The aim of this metaanalysis is to reassess the data of PORT on OS and DFS in stage III-N2 NSCLC, in order to figure out whether PORT might lead to a moderate improvement in local control and survival besides resection and adjuvant chemotherapy.

\section{Materials and methods}

Literature sources. A comprehensive search strategy was performed in the following electronic databases: EMBASE, PubMed, and Cochrane Library, for English language trials published from 1990 to July 2018, using the medical subject headings (Mesh) combined by (Carcinoma, Non-SmallCell Lung) AND (Surgery) AND (Chemoradiotherapy, Adjuvant) together with all other variable search terms. Reference records of relevant book chapters and publications were additionally searched. The proceedings of the most important international meetings in thoracic oncology were searched for studies published only as abstracts. Two reviewers independently evaluated the literatures, and screened titles and abstracts to identify potentially eligible articles. Any discrepancies were resolved by discussion and a third reviewer was consulted to achieve consensus when necessary. Results of the eligible articles are displayed in Table 1.

Inclusion and exclusion criteria. All available randomized controlled trials and retrospective comparative studies that compared the combination of PORT and adjuvant chemotherapy with adjuvant chemotherapy alone in resected N2 NSCLC were included. Editorials comments, letters to editors, conference abstracts, case reports, review studies and experimental cell or animal studies were excluded. The inclusion criteria include 1) studies on locally advanced or stage III N2 NSCLC patients who underwent adjuvant chemotherapy after surgical resection alone (no-PORT group) or that combined with PORT (PORT group), regardless the sequencing of chemotherapy and radiotherapy; 2) full texts and adequate data on OS, DFS (e.g., survival curves, hazard ratios and $95 \%$ confidence interval) for both the groups were available; 3 ) studies that were published and written in English.

Outcome measurement. Data associated with the following were extracted from the included articles: author names, year of publication, accrual period, patient source, study design, number of patients in the PORT group and the no-PORT group, disease stage, extent of resection, neo-adjuvant and adjuvant treatments, and data on OS and DFS of each group. The authors of the original articles were contacted for further information if relevant results were unclear.

The primary end point was OS and the secondary end point was DFS. For these time-to-event outcomes, the most appropriate statistic is the hazard ratio (HR) and associated 95\% confidence intervals [CI]. The HRs and associated 95\% CIs were extracted directly if offered in the results. Otherwise, they were estimated indirectly from other available statistics or from data extracted from reported Kaplan-Meier curves [19], using the statistical methods provided by Parmar et al. [20] and Tierney et al. [21].

We used the Newcastle-Ottawa Scale [22] to systematically assess the included retrospective studies. The methodological quality of included RCTs were assessed in terms of random sequence generation (selection bias), allocation concealment (selection bias), blinding of participants and personnel (performance bias), blinding of outcome assessment (detection bias), incomplete outcome data (attrition bias), selective reporting (reporting bias) and other bias by using the Cochrane collaboration's tool for assessing risk of bias [23]. Two authors independently performed the data extraction and statistical work and evaluated each article.Statistical Analysis. This meta-analysis was carried out according to the PRISMA guidelines and the Cochrane Collaboration Guidelines [24] and had registered in the international prospective register of systematic reviews (PROSPERO). 
Data were managed and analyzed using the RevMan software (Review Manager, version 5.3 for Windows). Statistically significant difference was set at a two-sided p-value less than 0.05 . The statistical heterogeneity among various studies was evaluated by a visual inspection of forest plots and quantified using $\mathrm{Chi}^{2}$ test, and the inconsistency was tested using $\mathrm{I}^{2}$ statistic. Randomeffects model was applied to hazard ratio (HR) analysis if the heterogeneity exists ( $\mathrm{I}^{2}$ values $>50 \%$, $\mathrm{p}$-value $<0.05$ ), otherwise fixed-effects model would be used. Subgroup analyses were further conducted according to study design and ethnicity.

\section{Results}

Overview of searching results. As shown in Figure 1, we identified 483 unique citations by the comprehensive search, of which 276 potentially relevant references were identified through initial title screening. Then 16 records were selected based on their titles, abstracts and article types, which were obtained as full-text articles and evaluated for inclusion and exclusion criteria. After full publication review, 11 studies [25-35] comparing PORT and no-PORT combined with adjuvant chemotherapy after surgical resection in stage III N2 NSCLC patients were finally included for evidence synthesis.

Characteristics of included studies. The characteristics of the studies included in this meta-analysis are summarized in Table 1. A total of 8,928 patients from 11 studies published from 2007 to 2017 were included, with 3,185 (35.67\%) patients receiving PORT plus adjuvant chemotherapy and 5,743 (64.33\%) undergoing adjuvant chemotherapy alone. Among the 11 studies, three of them were randomized-controlled trials [31, $33,34]$ (237 patients), four were multicenter retrospective studies $[27,28$, $32,35](7,748$ patients $)$ and four were single-center retrospective studies [25, 26, 29, 30] (907 patients); four studies were from United States of America (7,602 patients), and seven from Asia

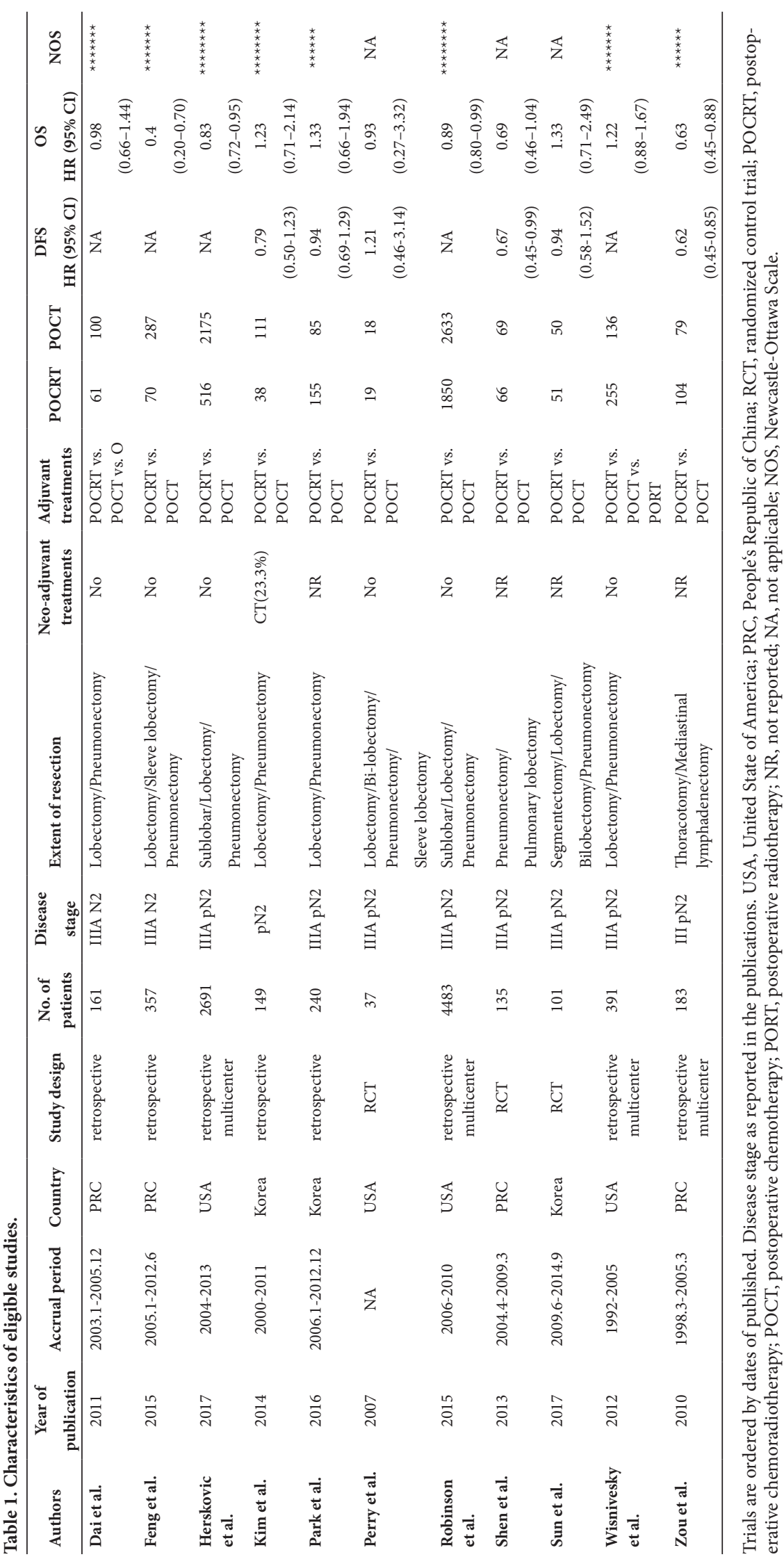




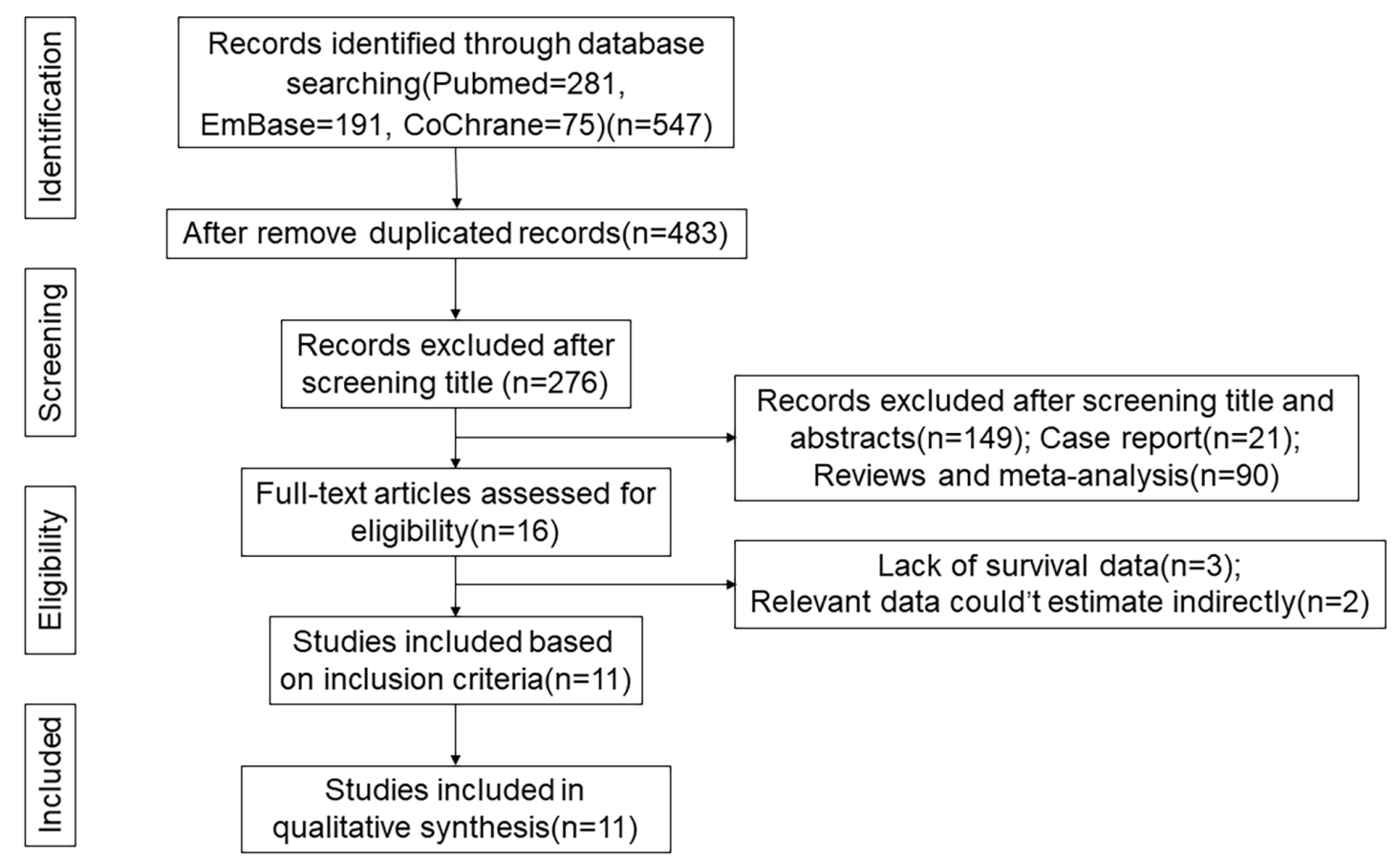

Figure 1. The flowchart of study identification procedure.

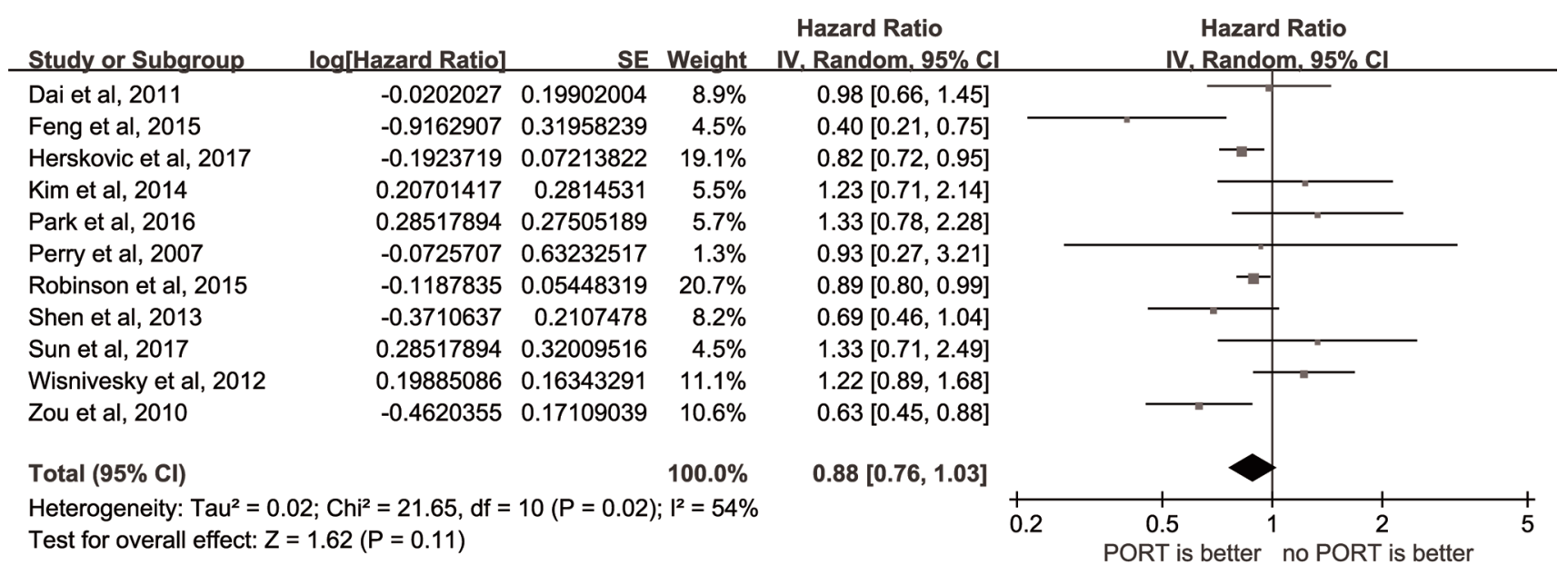

Figure 2. The forest plot of HRs for OS among the included studies.

(1,326 patients). All studies were sufficiently powered to evaluate OS and six studies were sufficiently powered to evaluate DFS [27, 29-31, 33, 34]. One study reported neo-adjuvant chemotherapy [29], five studies reported no neo-adjuvant treatment $[25,26,28,31,32,35]$ and four studies had no relevant content reported [27, 30, 33, 34]. Extent of resections comprised sublobar resection, lobectomy, bi-lobectomy, pneumonectomy, sleeve lobectomy, thoracotomy and mediastinal lymphadenectomy. Adjuvant treatments included PORT with adjuvant treatment, PORT alone, adjuvant treatment alone and observation.
Overall survival. Survival data were available for all 11 studies. For five studies [25, 27, 29-31], the p-values, number of events and the Kaplan-Meier curves were reported; and for the other six studies [26,28,32-35], the hazard ratio and confidence intervals were available. As shown in Figure 2, the combined results demonstrated a trend in improving survival associated with the use of PORT $(\mathrm{p}=0.11)$ with a hazard ratio of 0.88 ( $95 \% \mathrm{CI}, 0.76$ to 1.03 ); a moderate heterogeneity $\left(\mathrm{p}=0.02 ; \mathrm{I}^{2}=54 \%\right)$ between study results, suggesting that the studies were moderately similar to be combined, thus the random-effects model was chosen. 
Overall survival by ethnicity and study design. As shown in Figure $3 \mathrm{~A}$, there was a statistically significant benefit (HR=0.88; 95\% CI, 0.81 to 0.96 ; $\mathrm{p}=0.003$ ) on OS for PORT in the studies based on Caucasian patients, representing an overall $12 \%$ relative reduction in the risk of death.
Heterogeneity among 4 studies was not significant $(\mathrm{p}=0.19$; $\mathrm{I}^{2}=38 \%$ ), therefore, a fixed-model effect was used in this analysis. A trend in increased OS was also observed in Asian patients who received PORT ( $\mathrm{HR}=0.86$; $95 \% \mathrm{CI}, 0.64$ to 1.15 ; $\mathrm{p}=0.11$ ), as shown in Figure $3 \mathrm{~B}$. The heterogeneity test was

A

\begin{tabular}{|c|c|c|c|}
\hline Study or Subgroup & log[Hazard Ratio] & & E Weight \\
\hline Herskovic et al, 2017 & -0.1923719 & 90.07213822 & $33.8 \%$ \\
\hline Perry et al, 2007 & -0.0725707 & $\begin{array}{ll}7 & 0.63232517\end{array}$ & $0.4 \%$ \\
\hline Robinson et al, 2015 & -0.1187835 & $5 \quad 0.05448319$ & $59.2 \%$ \\
\hline Wisnivesky et al, 2012 & 0.19885086 & $6 \quad 0.16343291$ & $6.6 \%$ \\
\hline \multicolumn{4}{|c|}{$\begin{array}{l}\text { Heterogeneity: } \mathrm{Chi}^{2}=4.82, \mathrm{df}=3(\mathrm{P}=0.19) ; I^{2}=38 \% \\
\text { Test for overall effect: } Z=2.92(P=0.003)\end{array}$} \\
\hline \multicolumn{4}{|l|}{ B } \\
\hline Study or Subgroup & log[Hazard Ratio] & SE & Weight \\
\hline Dai et al, 2011 & -0.0202027 & 0.19902004 & $16.7 \%$ \\
\hline Feng et al, 2015 & -0.9162907 & 0.31958239 & $11.5 \%$ \\
\hline Kim et al, 2014 & 0.20701417 & 0.2814531 & $13.0 \%$ \\
\hline Park et al, 2016 & 0.28517894 & 0.27505189 & $13.2 \%$ \\
\hline Shen et al, 2013 & -0.3710637 & 0.2107478 & $16.1 \%$ \\
\hline Sun et al, 2017 & 0.28517894 & 0.32009516 & $11.5 \%$ \\
\hline Zou et al, 2010 & -0.4620355 & 0.17109039 & $18.0 \%$ \\
\hline Total $(95 \% \mathrm{Cl})$ & & & $100.0 \%$ \\
\hline \multicolumn{4}{|c|}{$\begin{array}{l}\text { Heterogeneity: } \mathrm{Tau}^{2}=0.10 ; \mathrm{Chi}^{2}=16.34, \mathrm{df}=6(P=0.01) ; \mathrm{I}^{2}=63 \% \\
\text { Test for overall effect: } Z=1.02(P=0.31)\end{array}$} \\
\hline
\end{tabular}

Hazard Ratio Hazard Ratio

IV, Fixed, $95 \% \mathrm{Cl}$

$0.82[0.72,0.95]$

$0.93[0.27,3.21]$

$0.89[0.80,0.99]$

$1.22[0.89,1.68]$

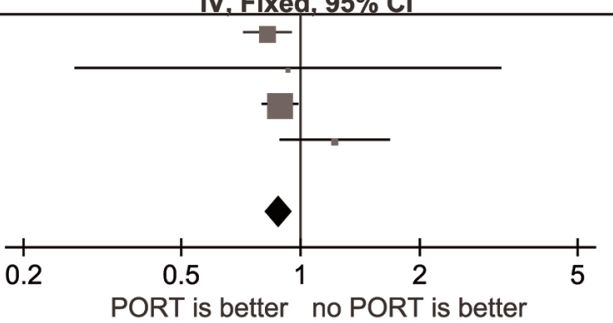

PORT is better no PORT is better

Hazard Ratio Hazard Ratio

Random, $95 \% \mathrm{Cl}$ IV. Random, 95\% C

$0.98[0.66,1.45]$

$0.40[0.21,0.75]$

$1.23[0.71,2.14]$

$1.33[0.78,2.28]$

$0.69[0.46,1.04]$

$1.33[0.71,2.49]$

$0.63[0.45,0.88]$

$0.86[0.64,1.15]$

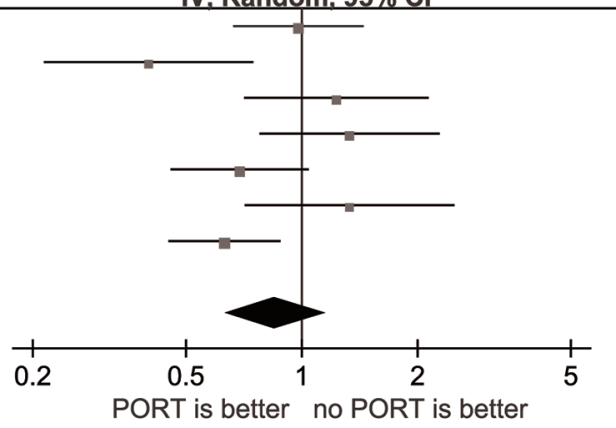

Figure 3. The forest plot of HRs for OS based on Caucasian patients (A), as well as based on Asian patients (B) among the included studies.

A

\begin{tabular}{lrr} 
Study or Subgroup & log[Hazard Ratio] & SE \\
\hline Perry et al, 2007 & -0.0725707 & 0.63232517 \\
Shen et al, 2013 & -0.3710637 & 0.2107478 \\
Sun et al, 2017 & 0.28517894 & 0.32009516 \\
& & \\
Total $(95 \%$ Cl) & \\
Heterogeneity: Chi $^{2}=2.96$, df $=2(P=0.23) ; I^{2}=32 \%$ \\
Test for overall effect: $Z=0.98(P=0.33)$
\end{tabular}

Hazard Ratio Hazard Ratio

IV. Fixed, $95 \% \mathrm{Cl}$

$7.2 \% \quad 0.93[0.27,3.21]$

$64.7 \% \quad 0.69[0.46,1.04]$

$28.1 \% \quad 1.33[0.71,2.49]$

$100.0 \% \quad 0.85[0.61,1.18]$

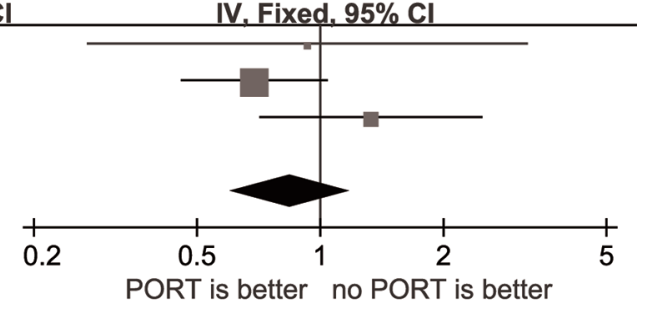

Hazard Ratio Hazard Ratio

Study or Subgroup log[Hazard Ratio] SE Weight IV, Random, 95\% Cl

Dai et al, $2011 \quad-0.0202027 \quad 0.19902004 \quad 10.5 \%$

Feng et al, 2015

Herskovic et al, 2017

Kim et al, 2014

Park et al, 2016

Robinson et al, 2015

Wisnivesky et al, 2012

Zou et al, 2010

$\begin{array}{rrr}-0.0202027 & 0.19902004 & 10.5 \% \\ -0.9162907 & 0.31958239 & 5.4 \%\end{array}$

$-0.1923719 \quad 0.07213822 \quad 21.7 \%$

$\begin{array}{lll}0.20701417 & 0.2814531 & 6.6 \%\end{array}$

$\begin{array}{lll}0.28517894 & 0.27505189 & 6.8 \%\end{array}$

$\begin{array}{lll}-0.1187835 & 0.05448319 & 23.3 \%\end{array}$

$0.19885086 \quad 0.16343291 \quad 13.1 \%$

$-0.4620355 \quad 0.17109039 \quad 12.5 \%$

Total $(95 \% \mathrm{Cl})$

$100.0 \%$

Heterogeneity: $\mathrm{Tau}^{2}=0.03 ; \mathrm{Chi}^{2}=18.67, \mathrm{df}=7(\mathrm{P}=0.009) ; \mathrm{I}^{2}=62 \%$

Test for overall effect: $Z=1.43(P=0.15)$
$0.98[0.66,1.45]$

$0.40[0.21,0.75]$

$0.82[0.72,0.95]$

$1.23[0.71,2.14]$

$1.33[0.78,2.28]$

$0.89[0.80,0.99]$

$1.22[0.89,1.68]$

$0.63[0.45,0.88]$

$0.89[0.75,1.05]$ IV, Random, $95 \% \mathrm{Cl}$

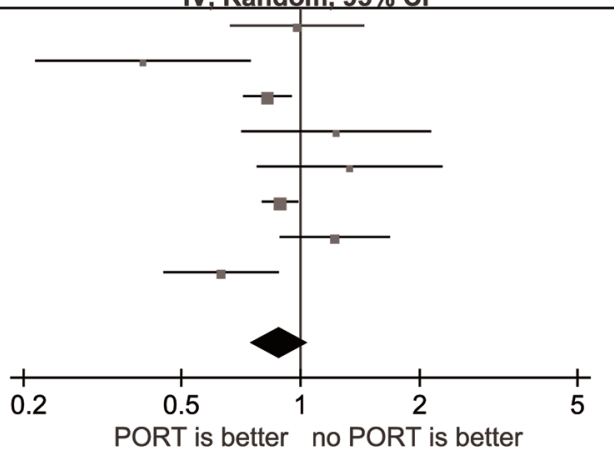

Figure 4. The forest plot of HRs for OS among the four RCT trials (A), as well as among retrospective studies (B). 
significant $(\mathrm{p}=0.01)$, and the $\mathrm{I}^{2}$ index indicated that $63 \%$ of the variability across trials was due to heterogeneity rather than chance, which led to use of random-effects model for analysis.

As shown in Figure 4A, significant heterogeneity among the four RCT trials was not found $\left(\mathrm{p}=0.23 ; \mathrm{I}^{2}=32 \%\right)$ and a fixed-model effect was used in further analysis. The combined HR of these trials was 0.85 (95\% CI, 0.61 to 1.18), which was not a statistically significant result $(\mathrm{p}=0.33)$ and favored PORT. As presented in Figure 4B, there was a moderate heterogeneity $\left(\mathrm{p}=0.009 ; \mathrm{I}^{2}=62 \%\right)$ among retrospective study results and the random-effect model was applied in these analyses. In addition, the effect of PORT on survival did not differ significantly with adjuvant chemotherapy alone ( $\mathrm{HR}=0.89$; 95\% CI, 0.75 to 1.05 ; $\mathrm{p}=0.15$ ).

Disease free survival. Disease free survival data were available for all 6 studies. For four studies [27,29-31,33], the $p$-value, number of events and the published curve were available; and for the other two studies [34] the hazard ratio and confidence intervals were available. As shown in Figure 5 , significant heterogeneity among the 6 trials was found with a p-value of 0.38 and the $\mathrm{I}^{2}$ index indicated that $6 \%$ of the variability across studies was due to heterogeneity rather than chance, resulting in the utilization of fixed-effects model for further analysis. We found significantly difference of effect on DFS between PORT combined with adjuvant chemotherapy and adjuvant chemotherapy alone $(\mathrm{HR}=0.78 ; 95 \% \mathrm{CI}, 0.66$ to $0.92 ; \mathrm{p}=0.003$ ).

Disease free survival by study design. As presented in Figure $6 \mathrm{~A}$, there was no significant heterogeneity $(\mathrm{p}=0.38$; $\left.\mathrm{I}^{2}=0 \%\right)$ among RCT trial results, suggesting that the trials were similar enough to be combined. The fixed-effects model was therefore chosen, and the combined results showed a trend in improving DFS associated with the use of PORT $(\mathrm{p}=0.13)$ with a hazard ratio of 0.80 (95\% CI, 0.60 to 1.07$)$. The heterogeneity test of 3 retrospective studies was not significant $(\mathrm{p}=0.19)$, the $\mathrm{I}^{2}$ index indicated that $40 \%$ of the variability across studies was due to heterogeneity rather

\begin{tabular}{lrrr} 
Study or Subgroup & log[Hazard Ratio] & SE & Weight \\
\hline Kim et al, 2014 & -0.2357223 & 0.229633 & $13.4 \%$ \\
Park et al, 2016 & -0.0618754 & 0.15961885 & $27.7 \%$ \\
Perry et al, 2007 & 0.19062036 & 0.48998765 & $2.9 \%$ \\
Shen et al, 2013 & -0.40047757 & 0.20113708 & $17.5 \%$ \\
Sun et al, 2017 & -0.0618754 & 0.24577488 & $11.7 \%$ \\
Zou et al, 2010 & -0.4780358 & 0.16224203 & $26.8 \%$ \\
& & & \\
Total (95\% Cl) & & $100.0 \%$ \\
Heterogeneity: Chi ${ }^{2}=5.32$, df = $5(P=0.38) ; I^{2}=6 \%$ & \\
Test for overall effect: $Z=2.96(P=0.003)$ &
\end{tabular}

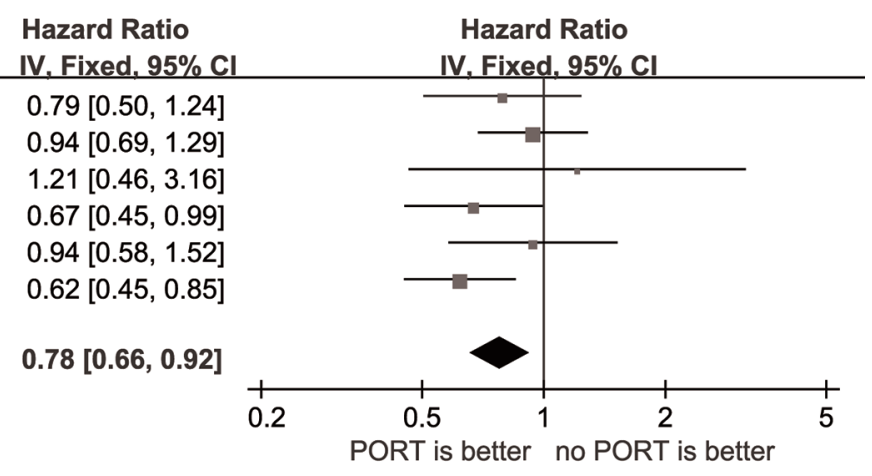

Figure 5. The forest plot of HRs for DFS among the included studies.

\section{A}

\begin{tabular}{|c|c|c|c|}
\hline Study or Subgroup & log[Hazard Ratio] & SE & Weight \\
\hline Perry et al, 2007 & 0.19062036 & 0.48998765 & $9.2 \%$ \\
\hline Shen et al, 2013 & -0.40047757 & 0.20113708 & $54.4 \%$ \\
\hline Sun et al, 2017 & -0.0618754 & 0.24577488 & $36.4 \%$ \\
\hline $\begin{array}{l}\text { Total }(95 \% \mathrm{Cl}) \\
\text { Heterogeneity: } \mathrm{Chi}^{2}= \\
\text { Test for overall effect }\end{array}$ & $\begin{array}{l}92, \mathrm{df}=2(P=0.38) \\
=1.50(P=0.13)\end{array}$ & $; l^{2}=0 \%$ & 100. \\
\hline $\begin{array}{l}\text { B } \\
\text { Study or Subgroup }\end{array}$ & log[Hazard Ratio] & SE & Weig \\
\hline Kim et al, 2014 & -0.2357223 & 0.229633 & $19.7 \%$ \\
\hline Park et al, 2016 & -0.0618754 & 0.15961885 & 40.8 \\
\hline Zou et al, 2010 & -0.4780358 & 0.16224203 & $39.5^{\circ}$ \\
\hline Total $(95 \% \mathrm{Cl})$ & & & 100.0 \\
\hline
\end{tabular}

Hazard Ratio Hazard Ratio

IV. Fixed. $95 \% \mathrm{Cl}$

$1.21[0.46,3.16]$

$0.67[0.45,0.99]$

$0.94[0.58,1.52]$

$0.80[0.60,1.07]$

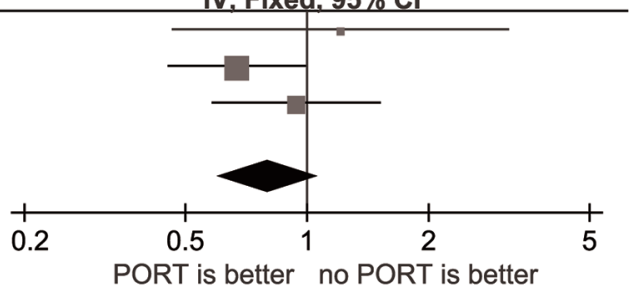

Hazard Ratio

IV. Fixed, $95 \% \mathrm{Cl}$

$0.79[0.50,1.24]$

$0.94[0.69,1.29]$

$0.62[0.45,0.85]$

$0.77[0.63,0.94]$

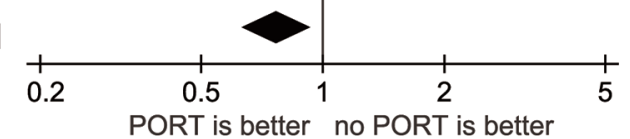

Figure 6. The forest plot of HRs for DFS among the four RCT trials (A), as well as among retrospective studies (B). 
than chance as shown in Figure 6B. Increased DFS was also observed in patients who received PORT with a significant combined HR of 0.77 ( $95 \%$ CI, 0.63 to $0.94 ; \mathrm{p}=0.01$ ).

Quality assessment. As summarized in Table 1, the eight eligible retrospective studies were systematically assessed by the Newcastle-Ottawa Scale, scored range from 6 to 8 out of 9 , and represented fairly high quality studies that led to less distortion of the summary effect estimate. The bias assessment of the three included RCTs were evaluated by the Cochrane collaboration's tool for assessing risk of bias as shown in Figure 7. There is no indication of significant bias among the included RCTs.

\section{Discussion}

Resectable, stage III-N2 NSCLC is related to a high risk of developing local recurrences and distant metastases. The poor prognosis after surgery had led to efforts to add postoperative treatments (chemotherapy and/or radiotherapy), hoping to optimize long-term outcomes by reducing the tumor progression. At present, there is sufficient evidence to demonstrate that adjuvant chemotherapy is expected to act as a standard treatment for patients with resected stage III-N2 NSCLC $[17,18]$. However, the evidence for application of PORT is still inconsistent. Our meta-analysis summarized all the studies regarding PORT on OS and DFS in stage III-N2 NSCLC. Based on the available published data, our results revealed that the use of PORT has a tendency to prolong OS $(\mathrm{HR}=0.88 ; 95 \% \mathrm{CI}, 0.76$ to $1.03 ; \mathrm{p}=0.11)$ and to significantly improve DFS (HR=0.78; 95\% CI, 0.66 to $0.92 ; \mathrm{p}=0.003)$. However non-significant and small the benefit of OS is, it is not surprising because this analysis involved only three RCTs and eight retrospective studies. The postoperative therapies included both sequential chemoradiotherapy and concurrent chemoradiotherapy and possibly selected patients for PORT, and the excess toxicity, particularly threatening cardiac and pulmonary toxicity together with non-cancer related deaths were also observed in these studies, which ineluctably led to uncertain conclusions. In fact, many studies demonstrated more benefit on survival with the application of PORT. A phase III study showed that compared with chemotherapy alone, PORT in addition to chemotherapy significantly improved 5-year survival to $47.4 \%$ (vs. 34\%) for pN2 disease, although this study was neither randomized nor mandatory [36]. Further, a recent updated meta-analysis of 16 trials comprising 3,278 patients indicated that PORT with modern techniques significantly improved locoregional recurrencefree survival, DFS and OS in patients with resectable stage IIIA-N2 NSCLC, with or without induction and/or adjuvant chemotherapy [37].

Considering that different ethnicities and study designs might result in different outcomes of OS and DFS, we analyzed the OS and DFS based on different subgroups. As shown in the results, there was a statistically significant
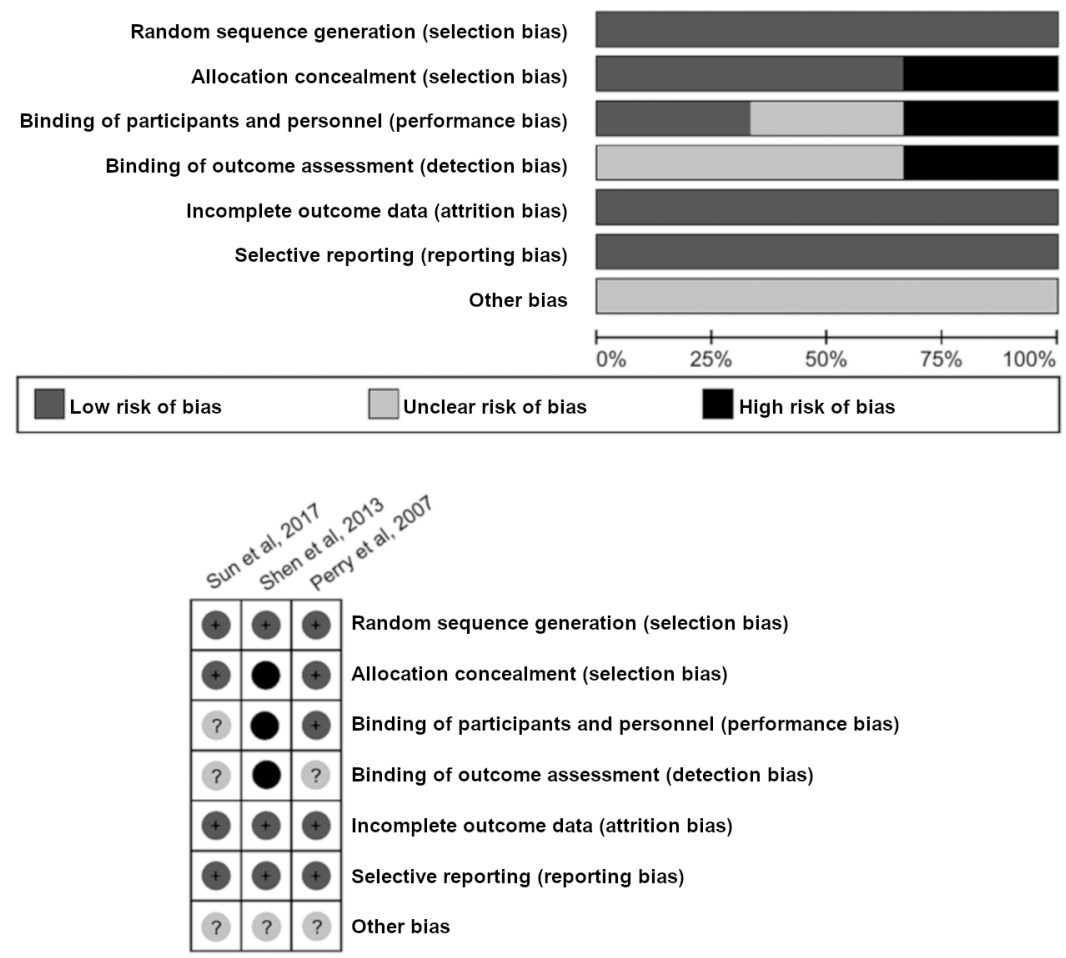

Figure 7. The bias assessment of the three included RCTs evaluated by the Cochrane collaboration's tool. 
benefit (HR=0.88; 95\% CI, 0.81 to $0.96 ; p=0.003$ ) on OS for PORT in the studies based on Caucasian patients, and a trend in increased OS was also observed in Asian patients who received PORT $(\mathrm{HR}=0.86$; 95\% $\mathrm{CI}, 0.64$ to $1.15 ; \mathrm{p}=0.11$ ) but the heterogeneity test was significant. However, the effect of PORT on survival did not differ significantly based on RCTs or retrospective studies. Compared with other studies [12,13], the effect of PORT on survival is similar. As for the local recurrence, which remained a crucial challenge with PORT. This meta-analysis demonstrated a trend in improving DFS associated with the use of PORT $(\mathrm{p}=0.13)$ with a hazard ratio of 0.80 (95\% CI, 0.60 to 1.07 ) and no significant heterogeneity $\left(\mathrm{p}=0.38 ; \mathrm{I}^{2}=0 \%\right)$ between RCT trial results, on the other hand, increased DFS was also observed in patients who received PORT with a significant combined HR of 0.77 (95\% CI, 0.63 to $1.18 ; \mathrm{p}=0.01$ ) from the three retrospective studies. Many hospitals used old suboptimal radiation techniques, and large treatment fields and Cobalt-60 radiation were criticized for being responsible for locoregional toxicity. In consequence, accompanying with the improvement of radiotherapy technologies such as standard fractionation, three-dimensional conformal radiation therapy (3DCRT), and intensity-modulated radiation (IMRT), the benefits of PORT were gradually shown up [38]. Previous meta-analysis have shown that the adverse effect of PORT is not significant for patients with stage III-N2 NSCLC [14]. Another meta-analysis of eleven phase III trials (2,387 patients) indicated that PORT utilization of linear accelerators decreased local recurrence with the most significant effect [39]. Further, the arrival of highly conformal IMRT greatly reduced the toxicity of PORT [40]. Dose escalation of PORT might reveal promising results for optimizing radiotherapy. Conventional radiation therapy already showed improvement of therapeutic effect in stage III NSCLC by increasing dosage, but toxicity was still a problematic issue to be addressed [41]. Via individualized, isotoxic, accelerated radiotherapy (INDAR), over 80 Gy biological doses could be administrated with toxicity levels comparable to $65 \mathrm{~Gy}$ [42]. Although the patients recovering from the adverse effects of surgical resection and adjuvant chemotherapy may be less tolerant to the toxicity of PORT, more evidences have demonstrated that PORT is related to improving both OS and DFS with lower toxicity by modern radiation techniques, helping patients with stage III-N2 NSCLC to complete the entire course of multimodality treatments.

It should be noted that our study has several limitations. First, the included studies are moderately heterogeneous in OS, which may affect the pooled estimation. Second, there are potential distortion of the summary effect estimate by retrospective studies and possible biases from participants, personnel and outcome assessment by RCTs, as well as the medium sample size of the latter. Third, no data in this study implies the relationship among extent of resections, incompletely resected types, and PORT techniques with clinical outcomes on survival. Finally, the III-N2 category was not a homogeneous group or the changes in stage III disease of 8th edition TNM classification applied from January 2017, so we could not predict which specific kind of patients might benefit from PORT.

We only concentrated on stage III-N2 NSCLC patients who had received chemotherapy with or without PORT after surgery to make sure we collected the appropriate data of interest for our study. This meta-analysis provides evidences that PORT is associated with improved OS and leads to a significantly increased DFS. Further exploration is needed to examine the effects of different radiotherapy techniques and doses on survival. More precise classification of patients and combined modality therapy will help answer some questions for the superior approach of resectable, stage III-N2 NSCLC.

Acknowledgements: This work was supported by grants from the Key Program of Heilongjiang Provincial Science and Technology Department (No. 2014003000017).

\section{References}

[1] JEMAL A, TIWARI RC, MURRAY T, GHAFOOR A, SAMUELS A et al. Cancer statistics, 2004. CA Cancer J Clin 2004; 54: 8-29. https://doi.org/10.3322/canjclin.54.1.8

[2] DECAMP MM Jr, ASHIKU S, THURER R. The role of surgery in N2 non-small cell lung cancer. Clin Cancer Res 2005; 11: 5033s-5037s. https://doi.org/10.1158/1078-0432.CCR05-9013

[3] LE CHEVALIER T, ARRIAGADA, PIGNON JP, SCAGLIOTTI GV. Should adjuvant chemotherapy become standard treatment in all patients with resected non-small-cell lung cancer? The Lancet Oncol 2005; 6: 182-184.

[4] SANTOS ES, CASTRELLON A, BLAYA M, RAEZ LE. Controversies in the management of stage IIIA non-small-cell lung cancer. Expert Rev Anticancer Ther 2008; 8: 1913-1929. https://doi.org/10.1586/14737140.8.12.1913

[5] ANDRE F, GRUNENWALD D, PIGNON JP, DUJON A, PUJOL JL et al. Survival of patients with resected N2 nonsmall-cell lung cancer: evidence for a subclassification and implications. J Clin Oncol 2000; 18: 2981-2989. https://doi. org/10.1200/JCO.2000.18.16.2981

[6] LORENT N, DE LEYN P, LIEVENS Y, VERBEKEN E, NACKAERTS K et al. Long-term survival of surgically staged IIIA-N2 non-small-cell lung cancer treated with surgical combined modality approach: analysis of a 7-year prospective experience. Ann Oncol 2004; 15: 1645-1653. https://doi. org/10.1093/annonc/mdh435

[7] JEREMIC B. Standard treatment option in stage III nonsmall-cell lung cancer: case against trimodal therapy and consolidation drug therapy. Clin Lung Cancer 2015; 16: 8085. https://doi.org/10.1016/j.cllc.2014.08.003

[8] LE PECHOUX C. Role of postoperative radiotherapy in resected non-small cell lung cancer: a reassessment based on new data. Oncologist 2011; 16: 672-681. https://doi. org/10.1634/theoncologist.2010-0150 
[9] SAYNAK M, HIGGINSON DS, MORRIS DE, MARKS LB. Current status of postoperative radiation for non-small-cell lung cancer. Semin Radiat Oncol 2010; 20: 192-200. https:// doi.org/10.1016/j.semradonc.2010.01.008

[10] LUNG CANCER STUDY GROUP. Effects of postoperative mediastinal radiation on completely resected stage II and stage III epidermoid cancer of the lung. N Engl J Med 1986; 315: 1377-1381. https://doi.org/10.1056/ NEJM198611273152202

[11] MAYER R, SMOLLE-JUETTNER FM, SZOLAR D, STUECKLSCHWEIGER GF, QUEHENBERGER $\mathrm{F}$ et al. Postoperative radiotherapy in radically resected non-small cell lung cancer. Chest 1997; 112: 954-959. https://doi. org/10.1378/chest.112.4.954

[12] LALLY BE, ZELTERMAN D, COLASANTO JM, HAFFTY BG, DETTERBECK FC et al. Postoperative radiotherapy for stage II or III non-small-cell lung cancer using the surveillance, epidemiology, and end results database. J Clin Oncol 2006; 24: 2998-3006. HTTPS://DOI.ORG/10.1200/ JCO.2005.04.6110

[13] WANG EH, CORSO CD, RUTTER CE, PARK HS, CHEN $\mathrm{AB}$ et al. Postoperative Radiation Therapy Is Associated With Improved Overall Survival in Incompletely Resected Stage II and III Non-Small-Cell Lung Cancer. J Clin Oncol 2015; 33: 2727-2734. https://doi.org/10.1200/JCO.2015.61.1517

[14] PORT META-ANALYSIS TRIALISTS GROUP. Postoperative radiotherapy in non-small-cell lung cancer: systematic review and meta-analysis of individual patient data from nine randomised controlled trials. Lancet (London, England) 1998 ; 352: 257-263.

[15] PORT META-ANALYSIS TRIALISTS GROUP. Postoperative radiotherapy for non-small cell lung cancer. The Cochrane database of systematic reviews 2005; 2: CD002142. https://doi.org/10.1002/14651858.CD002142.pub2

[16] BURDETT S, RYDZEWSKA L, TIERNEY JF, FISHER DJ. A closer look at the effects of postoperative radiotherapy by stage and nodal status: updated results of an individual participant data meta-analysis in non-small-cell lung cancer. Lung Cancer 2013; 80: 350-352. https://doi.org/10.1016/j. lungcan.2013.02.005

[17] ARRIAGADA R, BERGMAN B, DUNANT A, LE CHEVALIER T, PIGNON JP et al. Cisplatin-based adjuvant chemotherapy in patients with completely resected non-small-cell lung cancer. N Engl J Med 2004; 350: 351-360. https://doi. org/10.1056/NEJMoa031644

[18] PIGNON JP, TRIBODET H, SCAGLIOTTI GV, DOUILLARD JY, SHEPHERD FA et al. Lung adjuvant cisplatin evaluation: a pooled analysis by the LACE Collaborative Group. J Clin Oncol 2008; 26: 3552-3559. https://doi.org/10.1200/ JCO.2007.13.9030

[19] WILLIAMSON PR, SMITH CT, HUTTON JL, MARSON AG. Aggregate data meta-analysis with time-to-event outcomes. Stat Med 2002; 21: 3337-3351. https://doi. org/10.1002/sim.1303

[20] PARMAR MK, TORRI V, STEWART L. Extracting summary statistics to perform meta-analyses of the published literature for survival endpoints. Stat Med 1998; 17: 2815-2834.
[21] TIERNEY JF, STEWART LA, GHHERSI D, BURDEET S, SYDES MR. Practical methods for incorporating summary time-to-event data into meta-analysis. Trials 2007; 8: 16. https://doi.org/10.1186/1745-6215-8-16

[22] ZENG X, ZHANG Y, KWONG JSW, ZHANG C, LI S et al. The methodological quality assessment tools for preclinical and clinical studies, systematic review and meta-analysis, and clinical practice guideline: a systematic review. J Evid Based Med 2015; 8: 2-10. https://doi.org/10.1111/ jebm.12141

[23] HigGins JPT, ALTMAN DG, GOTZSCHE PC, JUNI P, MOHER D et al. The Cochrane Collaboration's tool for assessing risk of bias in randomised trials. BMJ 2011; 343: d5928. https://doi.org/10.1136/bmj.d5928

[24] CLARKE M, HORTON R. Bringing it all together: LancetCochrane collaborate on systematic reviews. Lancet 2001; 357: 1728. https://doi.org/10.1016/S0140-6736(00)04934-5

[25] DAI H, HUI Z, JI W, LIANG J, LU J et al. Postoperative radiotherapy for resected pathological stage IIIA-N2 nonsmall cell lung cancer: a retrospective study of 221 cases from a single institution. Oncologist 2011; 16: 641-650. https://doi.org/10.1634/theoncologist.2010-0343

[26] FENG W, ZHANG Q, FU XL, CAI XW, ZHU ZF et al. The emerging outcome of postoperative radiotherapy for stage IIIA(N2) non-small cell lung cancer patients: based on the three-dimensional conformal radiotherapy technique and institutional standard clinical target volume. BMC Cancer 2015; 15: 348. https://doi.org/10.1186/s12885-015-1326-6

[27] ZOU BG, XU Y, LI T, LI WH, TANG BX et al. A multicenter retrospective analysis of survival outcome following postoperative chemoradiotherapy in non-small-cell lung cancer patients with N2 nodal disease. Int J Radiat Oncol Biol Phys 2010; 77: 321-328. https://doi.org/10.1016/j. ijrobp.2009.05.044

[28] HERSKOVIC A, MAUER E, CHRISTOS P, NAGAR H. Role of Postoperative Radiotherapy in Pathologic Stage IIIA (N2) Non-Small Cell Lung Cancer in a Prospective Nationwide Oncology Outcomes Database. J Thorac Oncol 2017; 12: 302-313. https://doi.org/10.1016/j.jtho.2016.09.135

[29] KIM BH, KIM HJ, WU HG, KANG CH, KIM YT et al. Role of postoperative radiotherapy after curative resection and adjuvant chemotherapy for patients with pathological stage N2 non-small-cell lung cancer: a propensity score matching analysis. Clin Lung cancer 2014; 15: 356-364. https:// doi.org/10.1016/j.cllc.2014.05.005

[30] PARK H, OH D, AHN YC, PYO H, NOH JM et al. Role of Adjuvant Thoracic Radiation Therapy and Full Dose Chemotherapy in pN2 Non-small Cell Lung Cancer: Elucidation Based on Single Institute Experience. Cancer Res Treat 2017; 49: 880-889. https://doi.org/10.4143/crt.2016.442

[31] PERRY MC, KOHMAN LJ, BONNER JA, GU L, WANG XF, et al. A phase III study of surgical resection and paclitaxel/ carboplatin chemotherapy with or without adjuvant radiation therapy for resected stage III non-small-cell lung cancer: Cancer and Leukemia Group B 9734. Clin Lung Cancer 2007; 8: 268-272. https://doi.org/10.3816/CLC.2007.n.005 
[32] ROBINSON CG, PATEL AP, BRADLEY JD, DEWEES T, WAQAR SN et al. Postoperative radiotherapy for pathologic N2 non-small-cell lung cancer treated with adjuvant chemotherapy: a review of the National Cancer Data Base. J Clin Oncol 2015; 33: 870-876. https://doi.org/10.1200/ JCO.2014.58.5380

[33] SHEN W, JI J, ZUO Y, PU J, XU YM et al. Comparison of efficacy for postoperative chemotherapy and concurrent radiochemotherapy in patients with IIIA-pN2 non-small cell lung cancer: an early closed randomized controlled trial. Radiother Oncol 2014; 110: 120-125. https://doi.org/10.1016/j. radonc.2013.10.008

[34] SUN JM, NOH JM, OH D, KIM HK, LEE SH et al. Randomized Phase II Trial Comparing Chemoradiotherapy with Chemotherapy for Completely Resected Unsuspected N2Positive Non-Small Cell Lung Cancer. J Thorac Oncol 2017; 12: 1806-1813. https://doi.org/10.1016/j.jtho.2017.09.1954

[35] WISNIVESKY JP, HALM EA, BONOMI M, SMITH C, MHANGO G et al. Postoperative radiotherapy for elderly patients with stage III lung cancer. Cancer 2012; 118: 44784485. https://doi.org/10.1002/cncr.26585

[36] DOUILLARD JY, ROSELL R, DE LENA M, RIGGI M, HURTELOUP $P$ et al. Impact of postoperative radiation therapy on survival in patients with complete resection and stage I, II, or IIIA non-small-cell lung cancer treated with adjuvant chemotherapy: the adjuvant Navelbine International Trialist Association (ANITA) Randomized. Int J Radiat Oncol Biol Phys 2008; 72: 695-701. https://doi.org/10.1016/j. ijrobp.2008.01.044

[37] SAKIB N, LI N, ZHU X, LI D, LI Y et al. Effect of postoperative radiotherapy on outcome in resectable stage IIIA-N2 non-small-cell lung cancer: an updated meta-analysis. Nucl Med Commun 2018; 39: 51-59. https://doi.org/10.1097/ MNM.0000000000000764
[38] DENG JY, WANG C, SHI XH, JIANG GL, WANG Y et al. Reduced toxicity with three-dimensional conformal radiotherapy or intensity-modulated radiotherapy compared with conventional two-dimensional radiotherapy for esophageal squamous cell carcinoma: a secondary analysis of data from four prospective clinical trials. Dis Esophagus 2016; 29: 1121-1127. https://doi.org/10.1111/dote.12435

[39] BILLIET C, DECALUWE H, PEETERS S, VANSTEENKISTE J, DOOMS C et al. Modern post-operative radiotherapy for stage III non-small cell lung cancer may improve local control and survival: a meta-analysis. Radiother Oncol 2014; 110: 3-8. https://doi.org/10.1016/j. radonc.2013.08.011

[40] CHUN SG, HU C, CHOY H, KOMAKI RU, TIMMERMAN RD et al. Impact of Intensity-Modulated Radiation Therapy Technique for Locally Advanced Non-Small-Cell Lung Cancer: A Secondary Analysis of the NRG Oncology RTOG 0617 Randomized Clinical Trial. J Clin Oncol 2017; 35: 56-62. https://doi.org/10.1200/JCO.2016.69.1378

[41] MCGARRY RC. Integrating stereotactic body radiation therapy in stage II/III non-small cell lung cancer: is local control important? Expert Rev Anticancer Ther 2014; 14: 1419-1427. https://doi.org/10.1586/14737140.2014.948858

[42] DE RUYSSCHER D, VAN BAARDWIJK A, STEEVENS J, BOTTERWECK A, BOSMANS G et al. Individualised isotoxic accelerated radiotherapy and chemotherapy are associated with improved long-term survival of patients with stage III NSCLC: a prospective population-based study. Radiother Oncol 2012; 102: 228-233. https://doi. org/10.1016/j.radonc.2011.10.010 